\title{
A Novel MRI Texture Analysis of Demyelination and Inflammation in Relapsing-Remitting Experimental Allergic Encephalomyelitis
}

\author{
Yunyan Zhang ${ }^{1,2}$, Jennifer Wells ${ }^{2}$, Richard Buist ${ }^{3}$, James Peeling ${ }^{3}$, \\ V. Wee Yong ${ }^{2}$, and J. Ross Mitchell ${ }^{1,2}$ \\ ${ }^{1}$ Department of Radiology, University of Calgary, Calgary, Alberta, CA T2N 1N4 \\ \{yunyzhan, rmitch, vyong\}@ucalgary.ca \\ ${ }^{2}$ Department of Clinical Neurosciences, University of Calgary, Calgary, AB, CA T2N 1N4 \\ JELAQ lundbeck. com \\ ${ }^{3}$ Department of Radiology, University of Manitoba, Winnipeg, MB, CA R3T 2N2 \\ \{rbuist, jpeeling\}@ms.umanitoba.ca
}

\begin{abstract}
We have developed a novel multiscale localized image texture analysis technique, based upon the polar Stockwell Transform (PST). In this paper we characterized image texture in vivo using the PST in histologically verified lesion areas in T2-weighted MRI of an animal model of multiple sclerosis. Both high and low frequency signals, representing inflammation and demyelination, were significantly increased in pathological regions compared to normal control tissue. This suggests that this new local spatial-frequency measure of image texture may provide a sensitive and precise indication of disease activity.
\end{abstract}

\section{Introduction}

Multiple sclerosis (MS) is a chronic inflammatory demyelinating disorder of the central nervous system (CNS). This disease affects over one million people worldwide, and is the leading cause of disability in young adults. ${ }^{1}$ Magnetic resonance imaging (MRI) is a well-established imaging modality for diagnosis and monitoring MS.

The characteristic focal "plaques" in the CNS of MS patients are readily demonstrated as hyperintense areas on T2-weighted MRI. However, subtle diffuse pathological changes in normal appearing white matter $(\mathrm{NAWM})^{2}$ are difficult to detect using conventional MRI techniques. Nevertheless, these abnormalities are believed to play a pivotal role in the neurological impairments in patients with MS.

The infiltration of inflammatory cells, including macrophages, predominates the cascade tissue damage during MS pathogenesis. Infiltrated macrophages can be labeled by uptaking systemically administered ultra small particles of iron oxide (USPIO) via phagocytosis. ${ }^{3}$ USPIO loaded macrophages at sites of inflammation induce significant shortening of MRI T2 relaxation time, which leads to decreased signal intensity on T2-weighted MRI. ${ }^{4}$ Therefore, conventional MR imaging can be complemented by the tracking of macrophages in vivo in the study of MS.

We hypothesize that as tissue becomes abnormal, its MRI 'texture' will change. Image texture refers to a local characteristic pattern of image intensity that identifies a tissue. Texture analysis generates computer measures that quantitatively describe 
texture content in an image. A majority of published accounts have used statistical techniques, the co-occurrence matrix method $^{5}$ for instance, to analyze image texture in $\mathrm{MS}^{6-8}$, and other diseases. ${ }^{9}$ In particular, statistical texture analysis has been applied with promise to: distinguish active and inactive lesions; differentiate normal and pathological spinal cord; and, characterize therapeutic response in T2-weighted MRI in MS. However, current statistical methods are limited in detecting subtle abnormalities in the NAWM in MS. ${ }^{7}$

The polar Stockwell Transform (PST) is a new analysis technique that represents texture by its local spatial frequency. The PST combines features of Fourier and wavelet transforms to provide both spatial and spectral information throughout an image. $^{10,11}$ That is, it uses a frequency-dependent localizing Gaussian window to isolate the Fourier domain for sub-region analysis. Thus, the PST is capable of detecting subtle characteristics of image pattern and intensity. Indeed, previous MRI PST studies ${ }^{12}$ in MS showed that low spatial frequency content increased in an area of NAWM up to 30 days prior to lesion formation, and decreased when active lesions became inactive. The in-vivo human studies, however, were unable to provide histological correlation of the MRI texture changes.
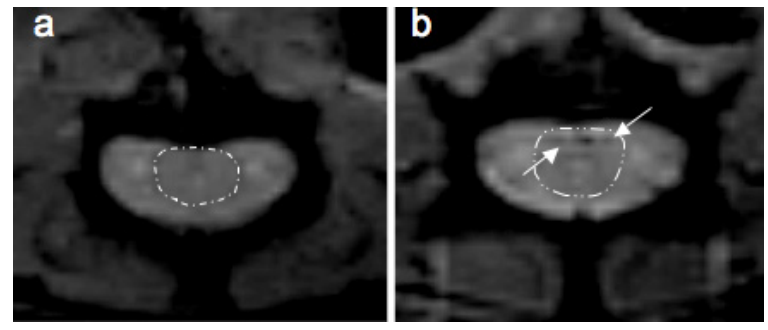

Fig. 1. Example $7 \mathrm{~T}$ T2-weighted MRI from a control (left) and an EAE (right) mouse. EAE cord (dashed oval) had higher signal intensity, larger volume than control cord. Two small low signal intensity lesions (arrows) were identified at the ventral side of lumber EAE cord.

The goal of the present study was to elucidate the histological substrate of image texture analyzed in the USPIO enhanced MRI from a murine model of MS, experimental allergic encephalomyelitis (EAE). The PST was applied to histologically defined lesion areas on $7 \mathrm{~T}$ T2-weighted MRI from the spinal cord of mice with EAE. This provided a unique opportunity to investigate local MRI spatial frequency content and compare that to tissue histopathology.

\section{Materials and Methods}

\subsection{Induction of EAE}

Eight to ten week old strain-129 mice were studied. EAE was induced in four mice by injecting subcutaneously $50 \mu \mathrm{g}$ of the immunogenic myelin oligodendrocyte glycoprotein (MOG) peptide (amino acids 35-55, synthesized by the Peptide Facility of the University of Calgary) emulsified in $100 \mu \mathrm{l}$ of complete Freund adjuvant (CFA) 
(Difco Laboratories, Detroit, Michigan). Pertussis toxin $(0.3 \mu \mathrm{g} / 200 \mu \mathrm{l}$, List Biological Laboratories, Campbell, Calif., USA) was injected intraperitoneally on the same day, and again two days later. EAE induced this way in stain-129 mice is characterized by relapsing-remitting episodes. Mice were weighed and evaluated daily. The degree of disability caused by EAE was assessed using neurological scores graded from 0 (normal) to 15 (death from EAE). Two age and strain matched non-EAE mice served as controls. All protocols for animal research conformed to the guidelines of the Animal Research Council of the Universities of Calgary and Manitoba as well as the Canadian Council on Animal Care.
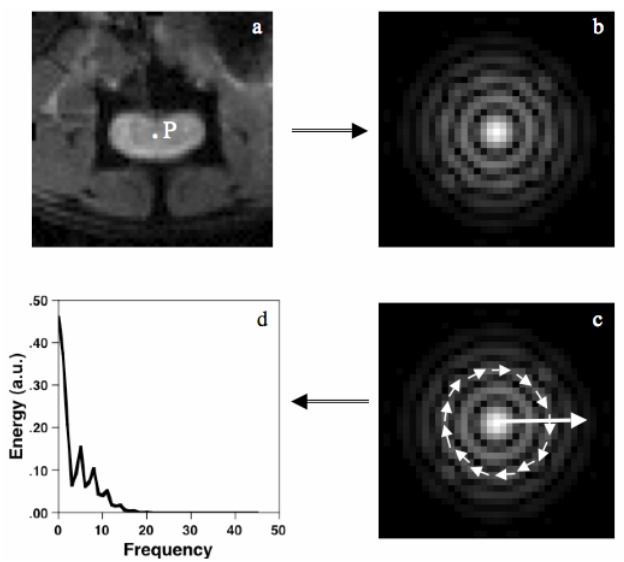

Fig. 2. The 1D spectral curve (d) was obtained by collapsing the 2D PST (b) calculated for pixel $P$ (a)

\subsection{MRI Experiments}

After induction of EAE, in vivo MR scans were performed at different time points, recorded as days post immunization (dpi). Two mice were imaged during remission of EAE (38 dpi and $21 \mathrm{dpi}$ ) and two were imaged at disease peak during the first relapse (35 dpi and 49 dpi). Inhalational isoflurane anesthesia (1.5\% in oxygen: $\left.\mathrm{N}_{2} \mathrm{O} 30: 70\right)$ was delivered via a nose cone during the imaging procedure. Each animal was positioned in a supine position within a $1.9 \mathrm{~cm}$ diameter birdcage coil. Respiration was monitored and the body temperature was maintained at $37^{0} \mathrm{C}$ by a thermocouple-based system during the MRI experiments.

The MRI examinations were performed on a $7 \mathrm{~T}$ Bruker horizontal bore NMR spectrometer. Ferumoxytol (AMI7228, Advanced Magnetics, Cambridge, MA) USPIO (30mg Fe/kg) was administered 24 hours before MRI scans to allow time for the USPIO-loaded macrophages to move into inflammation sites. T2-weighted MRI was acquired using an 8 -echo pulse sequence $(\mathrm{TR} / \mathrm{TE}=2540 \mathrm{~ms} / 26.79 \mathrm{~ms}$, matrix size $=256 \times 256, \mathrm{FOV}=2.5 \times 2.5 \mathrm{~cm}^{2}$, slice thickness $\left.=0.75 \mathrm{~mm}\right)($ Fig. 1$)$. This provided 12 interleaved slices covering the spinal cord from the lumbar (L2) to sacral segment (9 $\mathrm{mm}$ in length). For comparison, two control mice (without EAE, with USPIO) were also imaged using the same protocol. 


\subsection{Histopathology Examinations}

Immediately following the imaging experiment, the anesthetized animal was euthanized. The imaged spinal cord was carefully removed and immersed in $10 \%$ buffered formalin. Then the $9 \mathrm{~mm}$ long spinal cord segment was dissected into $61.5 \mathrm{~mm}$ blocks. Each of these blocks was then embedded in paraffin. Anatomical landmarks were strictly followed during this process to ensure the location of cord dissection matched with MRI slice position. Two continuous axial $6 \mu \mathrm{m}$ thick sections were cut every $0.75 \mathrm{~mm}$ and placed on separate glass slides. Two series of 12 sections each were prepared per animal, for a total of 144 slide preparations ([4 EAE +2 control] mice $\mathrm{x} 2$ series $\mathrm{x} 12$ sections). The first series of sections was stained with hematoxylin, eosin, and luxol fast blue for evidence of inflammation and demyelination. The second series was examined for the presence of macrophages and USPIO co-localized by Iab-1 immunostain and Prussian blue.

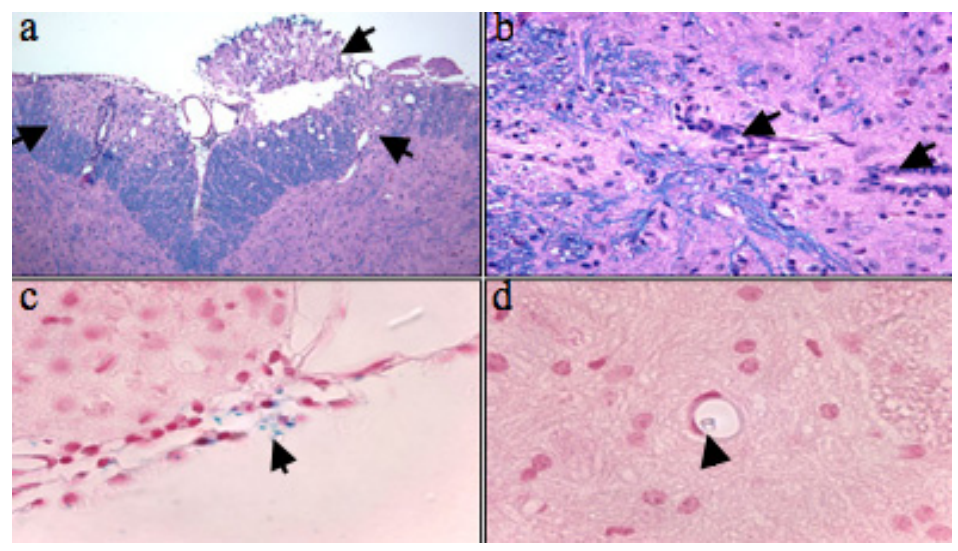

Fig. 3. Histology of the MRI lesion ROI at Fig. 1. Shown are demyelination (arrows, a), inflammation (arrows, b), and Prussian blue positive macrophages in the glia limitans (arrow, c) and in the blood vessel (arrow, d).

\subsection{PST Texture Analysis}

The polar Stockwell Transform of an image $I(x, y)$ is defined as follows: ${ }^{10}$

$$
S\left(x, y, k_{x}, k_{y}\right)=\int_{-\infty}^{+\infty} \int_{-\infty}^{+\infty} I\left(x^{\prime}, y^{\prime}\right) w\left(x^{\prime}-x, y^{\prime}-y, f, \theta\right) e^{-i 2 \pi\left(k_{x} x^{\prime}+k_{y} y^{\prime}\right)} d x^{\prime} d y^{\prime}
$$

Where, $w(x, y, f, \theta)$ is the localizing Gaussian window

$$
w(x, y, f, \theta)=\frac{k^{2} \lambda(k)}{2 \pi} e^{-\frac{k^{2}}{2}(x \cos \theta+y \sin \theta)^{2}} e^{-\frac{(k \lambda(k))^{2}}{2}(-x \sin \theta+y \cos \theta)^{2}}
$$


Here, $k=\sqrt{k_{x}^{2}+k_{y}^{2}}$ is the centre frequency, and $\theta=a \tan \left(\frac{k_{y}}{k_{x}}\right)$ is the frequency orientation. The Gaussian window is inversely proportional to the center frequency, $k$, and is rotated to the direction $\theta$. The PST is rotationally invariant, and therefore eliminates the effect on texture measurements of patient or image rotation.

The obtained MR images were non-uniformity corrected prior to any image processing using a polynomial algorithm developed in house. This process removed the possible intensity bias caused by the high field MR system and greatly improved the MRI contrast. Lesion-guided analysis was employed to determine the texture characteristics of pathological tissues. That is, texture was only analyzed from the low signal intensity areas on T2-weighted MRI when pathological changes at the same location were present on the spinal cord sections at the corresponding MRI slice position.

The PST spectrum of each ROI (32x32 pixels) was computed to first generate a local 2D Fourier spectrum for each pixel. Each local 2D spectrum was then reduced to a local 1D spectrum by integrating it along rings of constant width $\left(0.32 \mathrm{~cm}^{-1}\right)$ in the Fourier domain (Fig. 2). The local 1D spectra from the central 2x2 pixels in each ROI were averaged for analysis. An estimate of the low frequency energy within each ROI was obtained by summing the area under the local 1D spectrum below frequency $=$ $3.2 \mathrm{~cm}^{-1}$. The same ROIs in the corresponding MRI slices from normal mice were selected, and the 1D PST spectrum was calculated to obtain control values.

\subsection{Statistical Analysis}

The paired Student $t$-test and statistical software program (SPSS 11 for Macintosh) were used to compare MRI texture from EAE mice and from normal control mice. A $P$ value of less than 0.05 was considered significant.

\section{Results}

\subsection{MRI-Matched Neuropathology of Mice with EAE}

The mice imaged during remission started to show behavioral evidence of neuropathology at $12 \mathrm{dpi}$ and $14 \mathrm{dpi}$. Their disability scores on the date of MRI were 1 and 2 respectively. The EAE signs of the other two mice started relatively late ( $23 \mathrm{dpi}$ and $34 \mathrm{dpi}$ ), but the disability progressed fast such that they had disability scores of 9 and 7.5 when MRI was performed.

Eight ROIs in T2-weighted MR images, acquired during symptom remission, were matched with histopathology. Within these ROIs, there was a mixture of inflammation and demyelination, with the former dominating (Fig. 3, a and b). Prussian blue positive cells (monocytes and macrophages) were detected in the cord parenchyma, blood vessels and glia limitans (Fig. 3, c and d). The EAE mice imaged during disease peak had cellular infiltration throughout the spinal cord cross-sections. Consequently, focal lesions were not visible and could not be matched to the MRI ROIs. 


\subsection{Lesion Texture Changes Compared to Normal Control Tissue}

The spectral distributions were more variable in lesion ROIs than in normal tissue ROIs. However, all spectra had similar distributions showing greater energy at the lower frequency range $\left(0<\mathrm{f} \leq 3.2 \mathrm{~cm}^{-1}\right)$ than at the higher frequencies $(3.2<\mathrm{f} \leq 6.72$ $\mathrm{cm}^{1}$ ) (Fig. 4). The mean spectral distribution from lesion regions also had a similar shape but with increased energy compared to that from normal spinal cord areas.
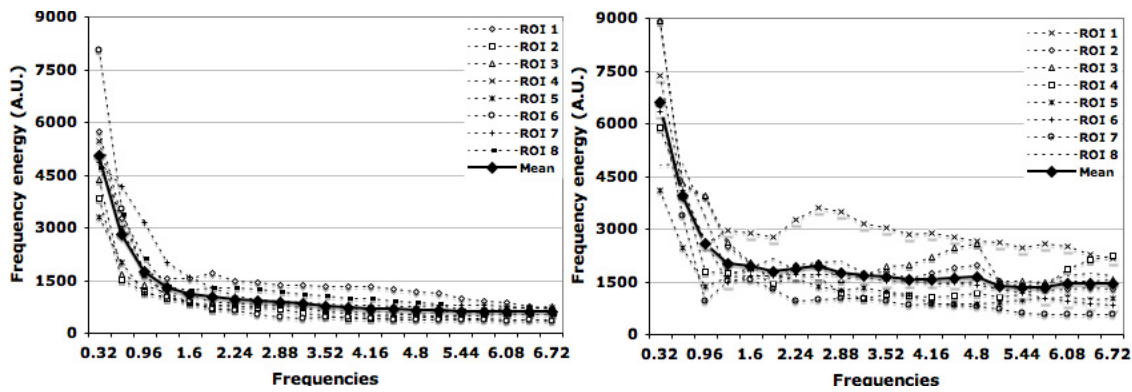

Fig. 4. Individual spectral distribution pattern and the mean spectrum of all ROIs from control (left) and EAE (right) mice quantified by the PST technique on T2-weighted MRI

The mean total energy at all spatial-frequencies was significantly higher $(\mathrm{p}<0.01)$ in lesion ROIs than in the corresponding normal tissue ROIs. The ratio of high to low frequency power was 0.63 in lesions (low, high frequency was $61.5 \%$ and $38.5 \%$ ) and 0.43 in control spinal cord (low, high frequency was $69.7 \%$ and $30.3 \%$ ). Further, both the low and high frequency energy was significantly increased $(\mathrm{p}<0.01)$ in diseased areas compared to normal control data (Fig. 5).

\section{Discussions}

This study tested the hypothesis that MRI texture, quantified by the novel local multiscale texture analysis, changes following tissue pathology. In the present study, the histological pathology was considered as the gold standard for locating the particular MRI abnormalities for texture analysis.

This study revealed that histopathological changes in EAE lesions corresponded to a significant increase in the local spectral energy determined by the PST, compared to controls. To our knowledge, this is the first study correlated tissue histopathology with MRI texture directly in vivo. A previous study, ${ }^{13}$ using statistical texture analysis, detected remyelination in serial 4.7 T T2-weighted MR images during 2-month disease course, in a demyelinating-remyelinating cuprizone mouse model. However, no histological analysis was performed in these experiments. Instead, the authors relied upon results of the cuprizone model in the literature to infer myelin integrity for comparison. Consequently, direct correlation between image texture and tissue pathology was not possible.

Generally, low frequency components correspond to large spatial scale and coarse texture. High frequency components correspond to small spatial scale and fine 
texture. Inflammatory cells, damaged myelin and fragments, and possibly axonal transection (data not shown), increased the hybridism of tissue structure. This may cause an unequal signal intensity change on T2-weighted MRI and a change in image texture. The elevated energy of the low frequency components suggests a coarse texture change in the affected tissue. On the other hand, inflammation usually accompanies edema, which first enlarges the EAE cord volume. ${ }^{14}$ Secondly, the increased extracellular water space may smooth away the uniqueness of tissue structure. Hence the tissue becomes finer that resulted in, at least partially, increased high frequency content.

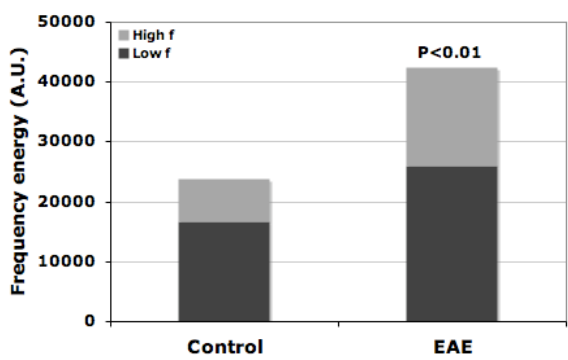

Fig. 5. Mean total spectral energy in EAE was significantly greater than that in normal tissue. The low frequency energy in pathological regions was also significantly greater than in control regions, so does the high frequency energy

Inflammatory cell infiltration is the earliest event in MS lesion initiation. ${ }^{1,3}$ Each of the lesion ROIs examined in this study contained variable degrees of inflammation including cellular infiltration. These pathological changes, however, do not always cause visible changes in T2-weighted MRI. ${ }^{15}$ In contrast, our new texture measure was significantly elevated in all ROIs where pathological changes were noted, even those with subtle and small histological changes. These findings are similar to those of a previous PST study in T2-weighted MRI in MS lesions, where the local frequency energy increased significantly in NAWM one month before lesion visualization. ${ }^{12}$ This suggests that the PST is sensitive to subtle texture changes even in the early stage of lesion formation.

Cellular infiltration inevitably causes subsequent demyelination during disease process. As a consequence, almost all the histology sections contained a mixture of inflammation and demyelination. This indicates that inflammation and demyelination each contributed to the spectral energy increase in lesion ROIs. Therefore, the PST analysis is nonspecific. However, the existence of either pathology in the tissue may induce texture changes in MRI, which can be readily captured by the PST.

A few MR imaging techniques have emerged in recent years to detect subtle histopathological changes in pre-lesion areas in normal appearing brain tissue but none of them is conclusive. The PST has been shown of potential to be a sensitive technique for MRI texture analysis in MS. Therefore, determining the histopathological correlates underlying PST texture is critical for its precise interpretation and accurate application, particularly in longitudinal MS studies.

In summary, this study showed that the pathological changes in an animal model of MS caused significant increase in both low and high frequency energy characterized by the PST. In this paper, a direct correlation of tissue histology with a PST analysis 
of MRI texture was used. It provided insight into the histopathological substrates causing local MRI spatial-frequency changes in MS. Hence it is of importance in directing future texture analysis in MS using the PST. Because of its sensitivity to image intensity and pattern changes, the PST may become a powerful tool to characterize subtle abnormalities in NAWM prior to lesion formation in MS. Furthermore, it may also aid in predicting early therapeutic responses in MS clinical trials.

\section{Acknowledgements}

We thank Mr. Brian O Brien for the development of MRI non-uniformity correction algorithm, and Dr. Hongmei Zhu for the initial implementation of the PST program.

\section{References}

1. I. Pirko, J. Gamez, A. J. Johnson, S. I. Macura, and M. Rodriguez. Dynamics of MRI lesion development in an animal model of viral-induced acute progressive CNS demyelination. NeuroImage 2004, 21: 576-582.

2. D. H. Miller, A. J. Thompson, M. Filippi. Magnetic resonance studies of abnormalities in the normal appearing white matter and grey matter in multiple sclerosis. J Neurol. 2003, 250: $1407-1419$.

3. S. Floris, E. L. A. Blezer, G. Schreibelt, E. Dopp, et al. Blood-brain barrier permeability and monocyte infiltration in experimental allergic encephalomyelitis. A quantitative study. Brain, 2004, 127: 1-12.

4. V. Dousset. In vivo Macrophage activity imaging in the central nervous system detected by magnetic resonance. MRM 1999, 41: 329-333.

5. R. M. Haralick, K. Shanmugam, and J. Dinstein. Texture features for image classification. IEEE Trans Syst Man Cybern. 1973, 6: 610-621.

6. O. Yu, Y. Mauss, G. Zollner, I. J. Namer, J. Chambron. Distinct patterns of active and non-active plaques using texture analysis on brain NMR images in multiple sclerosis patients: preliminary results. MRI 1999, 17: 1261-1267.

7. J. M. Mathias, P. S. Tofts. N. A. Losseff. Texture analysis of spinal cord pathology in multiple sclerosis. MRM 1999, 42: 929-935.

8. Y. Zhang, H. Zhu, R. Ferrari, W. Wei, et al. Texture analysis of MR images of minocycline treated MS patients. MICCAI proceeding 2003, 2878: 786-793.

9. R. A. Lerski, K. Straughan, L. R. Schad, D. Boyce, et al. MR image texture analysis - an approach to tissue characterization. MRI 1993, 11: 873-887.

10. R. G. Stockwell, L. Mansinha, and R. P. Lowe. Localization of the complex spectrum: The S transform. IEEE Trans. Signal Process. 1996, 44: 998-100.

11. H. Zhu, B. G. Goodyear, M. L. Lauzon, R. A. Brown, et al. A new local multiscale Fourier analysis for medical imaging. Med. Phys. 2003, 30: 1134-1141.

12. Y. Zhang, H. Zhu, L. M. Metz, J. R. Mitchell. A new MRI texture measure to quantify MS lesion progression. 12th ISMRM proceedings, 2004.

13. O. Yu, J. Steibel, Y. Mauss, B. Guignard, B. Eclancher, et al. Remyelination assessment by MRI texture analysis in a cuprizone mouse model. MRI 2004, 22: 1139-1144.

14. Y. Zhang, J. Wells, R. Buist, X. Sun, et al., "In vivo MRI quantification of mouse spinal cord with Relapsing Remitting EAE: Disability and imaging correlates," 13th ISMRM proceedings, 2005.

15. L. L. Cook, P. J. Foster, J. R. Mitchell, and S. J. Karlik. In vivo 4.0-T magnetic resonance investigation of spinal cord inflammation, demyelination, and axonal damage in chronicprogressive experimental allergic encephalomyelitis. J MRI 2004, 20: 563-571. 